\section{Crowded optic nerve head evaluation with optical coherence tomography in anterior ischemic optic neuropathy}

\author{
Abstract \\ Purpose To characterize the optic nerve \\ head $(\mathrm{ONH})$ structure in patients with \\ non-arteritic anterior ischemic optic \\ neuropathy (NAION) compared to healthy \\ control subjects using spectral domain \\ optical coherence tomography (SD-OCT) \\ via the enhanced depth imaging \\ method.
}

Methods In this prospective, cross-sectional, comparative study, we assessed 66 eyes of 33 patients with unilateral NAION and 31 eyes of 31 healthy normal subjects in an academic institution. The peripapillary nerve fiber layer thickness, disc area, and quantitative parameters of the ONH structures, including the Bruch's membrane opening (BMO) area, anterior laminar depth, and prelaminar thickness and depth were compared between the three groups.

Results Linear mixed model analysis after adjusting for age, sex, and axial length showed that the BMO area was similar in eyes with NAION $\left(1.89 \pm 0.33 \mathrm{~mm}^{2}\right)$, their fellow eyes $\left(1.85 \pm 0.35 \mathrm{~mm}^{2}\right)$, and control eyes $\left(1.88 \pm 0.37 \mathrm{~mm}^{2}\right.$; all $\left.P>0.99\right)$. Anterior laminar depth was also similar in the three groups. The mean prelaminar tissue thickness of the NAION eyes was $445 \pm 176 \mu \mathrm{m}$, which was thinner than the prelaminar tissue of their unaffected fellow eyes (mean, $539 \pm 227 \mu \mathrm{m}, P=0.004$ ), but both were thicker than the prelaminar tissue of the normal subjects (mean $243 \pm 145 \mu \mathrm{m}, P=0.001$ and $P<0.001$, respectively).

Conclusions The thick prelaminar thickness is associated with unilateral NAION in the affected and unaffected eyes.

Eye (2017) 31, 1191-1198; doi:10.1038/eye.2017.56; published online 7 April 2017
S Moghimi ${ }^{1,2}$, M Afzali ${ }^{1}$, M Akbari KB Ebrahimi ${ }^{3}$, A Khodabande ${ }^{1}$,

AR Yazdani-Abyaneh ${ }^{4}$, SNH Ghafouri ${ }^{1}$, P Coh ${ }^{2}$, S Okhravi ${ }^{1}$ and MA Fard ${ }^{1}$

\section{Introduction}

Non-arteritic anterior ischemic optic neuropathy (NAION) is the most common clinical presentation of acute ischemic damage to the optic nerve and is characterized by a sudden painless visual loss, optic disc edema, and peripapillary hemorrhage. ${ }^{1}$ Although the detailed cause of NAION is unclear, previous studies have found a clinically smaller optic disc area, a crowded optic nerve, and a smaller cupto-disc ratio in the unaffected fellow eyes of patients with unilateral NAION when compared to control eyes. ${ }^{2-5}$ A small scleral canal opening and Bruch's membrane opening (BMO), and an abnormally stiff lamina cribrosa (LC) are also hypothetically considered as the main risk factors for the development of NAION. ${ }^{6}$

Spectral domain optical coherence tomography (SD-OCT) with enhanced depth imaging (EDI) can reliably capture highresolution images of the deep optic nerve in glaucoma. There are fundamental differences between the clinical examination of the optic disc and SD-OCT imaging of the optic nerve head $(\mathrm{ONH}) \cdot{ }^{7-12}$ Our previous observation has shown that $\mathrm{ONH}$ in glaucoma patients is characterized by LC thinning compared to NAION eyes. ${ }^{12}$ A recent study has reported prelaminar tissue thickening and BMO enlargement in the acute edematous NAION eyes compared with unaffected eyes, with reversal of changes during follow-up. ${ }^{13}$

The morphologic characteristics of the optic disc before the onset of NAION could provide further information regarding the possible underlying predisposing factors through investigating the unaffected fellow eyes compared to control healthy eyes. Therefore, we measured the peripapillary retinal nerve fiber layer ( $p R N F L$ ) and ONH quantitative
${ }^{1}$ Farabi Eye Hospital, Eye Research Center, Tehran University of Medical Science, Tehran, Iran

${ }^{2}$ Koret Vision Center, University of California, San Francisco, CA, USA

${ }^{3}$ Mason Eye Institute, Columbia, MO, USA

${ }^{4}$ Noor Ophthalmology Research Center, Noor Eye Hospital, Tehran, Iran

Correspondence: MA Fard, Farabi Eye Hospital, Eye Research Center, Tehran University of Medical Science, South Kargar Street, Qazvin SQ, Tehran 13352, Iran Tel/Fax: +98 2155400003. E-mail: masood219@ gmail.com

Received: 20 April 2016 Accepted in revised form: 12 February 2017 Published online: 7 April 2017 
parameters, including the anterior LC depth, prelaminar tissue thickness, $\mathrm{BMO}$ distance, and $\mathrm{BMO}$ area in patients with chronic unilateral NAION, their unaffected fellow eyes, and healthy normal subjects using the SpectralisOCT software and compared the results among these groups.

\section{Material and methods}

\section{Subjects}

Patients with chronic unilateral NAION and age-matched healthy control subjects who were visited at the outpatient clinic of Farabi Eye Hospital between July 2014 and October 2015 were enrolled in this prospective, crosssectional, comparative study. The study was approved by the Ethics Committee of Tehran University of Medical Science and all investigations adhered to the tenets of the Declaration of Helsinki. Written informed consent was obtained from each participant after receiving a detailed explanation of the nature and objective of the study.

All the subjects underwent a thorough ophthalmic evaluation, including slit-lamp biomicroscopy, bestcorrected visual acuity (BCVA) using a LogMAR chart, refractive error examination with an autorefractometer, intraocular pressure (IOP) by Goldman applanatation tonometry, fully dilated fundus examination with defining clinically cup-to-disc ratio, as well as axial length measurement using ocular biometry (IOL Master, Carl Zeiss Meditec, Dublin, CA, USA). Perimetry was performed using the standard Swedish Interactive Thresholding Algorithm (SITA) with the 24-2 pattern on the Humphrey Field Analyzer (Carl Zeiss Meditec). Only reliable results were included.

The patients also underwent $\mathrm{ONH}$ and RNFL imaging using EDI SD-OCT (Specteralis, HEYEX software 6.0 Heidelberg Engineering, Heidelberg, Jena, Germany).

The inclusion criteria for unilateral NAION were a past history of sudden, painless visual loss in one eye together with optic disc swelling and/or superficial hemorrhage on the border of the disc or adjacent retina that occurred $>3$ months ago (by confirming the objective signs, or by asking the referring ophthalmologists), and an ophthalmologically healthy fellow eye. At the time of the study, optic disc swelling had subsided, and disc borders were sharp and discrete. The exclusion criteria for NAION patients were having an ocular or neurologic disease other than NAION, especially possible evidence of glaucoma, bilateral NAION, acute NAION, evidence of arteritic AION (systemic manifestations of giant cell arteritis, an erythrocyte sedimentation rate $>50 \mathrm{~mm} / \mathrm{h}$ and a positive C-reactive protein) or inflammatory optic neuritis.
The control group comprised age-matched subjects with a best-corrected visual acuity of $\geq 20 / 30$, intraocular pressure of $\leq 21 \mathrm{~mm} \mathrm{Hg}$, an open angle, normal optic disc appearance on fundus examination, and no visual field or pRNFL defects.

In all groups, patients below 18 years of age and those with refractive errors $\geq+6.00$ or $\leq-6.00 \mathrm{D}$ or more than $\pm 3.00 \mathrm{D}$ astigmatism, a history of ocular surgery (except for uncomplicated cataract surgery), or a glaucomatous or neurological disease were also excluded.

\section{Optical coherence tomography measurements}

Images were obtained using EDI-OCT after pupil dilation, and viewed and measured with the Heidelberg Eye Explorer software. Images with poor centration, segmentation errors, or poor quality $(<15 \mathrm{~dB})$ were excluded from analysis. Two sets of scans were obtained for each eye: pRNFL and ONH.

The measurement of the pRNFL was performed using the standard $360^{\circ}, 3.4 \mathrm{~mm}$ diameter circular scan and the mean PRNFL values were recorded.

For the ONH scan, the EDI-OCT device was set to image a $15 \times 15$-degree rectangle centered on the optic disc. This rectangle was divided into approximately 73 sections and each one had 42 OCT frames on average. From these horizontal B-scans, 3 frames (center, midsuperior and mid inferior) that passed through the $\mathrm{ONH}$ were selected and the anterior laminar depth (ALD) was measured in each of these frames. Prelaminar tissue thickness and prelaminar depth were measured only from the central ONH frame. The BMO was defined as the termination of the Bruch's membrane (one point on each side of the B-scan) and the line connecting both ends of the Bruch's membrane was defined as the BMO distance (Figure 1). The BMO area was the area created by connecting each side of several BMO distance lines. Laminar position measurements were made as close to the line perpendicular from the midpoint of the $\mathrm{BMO}$ reference plane as possible. When a vessel trunk made it difficult to take measurements (especially on the nasal side of disc), the measurements were performed on the temporal side.

The distance between the internal limiting membrane of the optic nerve head and the midpoint of the BMO was labeled the prelaminar depth. When the internal limiting membrane dipped below the BMO, we used plus sign. A minus sign for prelaminar depth description indicated elevation of the tissue from the BMO. The arbitrary plus or minus sign was used to differentiate depression and elevation of the ONH surface. The LC was identified as a highly reflective plate-like structure after adjusting the brightness and contrast of the images. The prelaminar thickness was defined as the perpendicular distance 

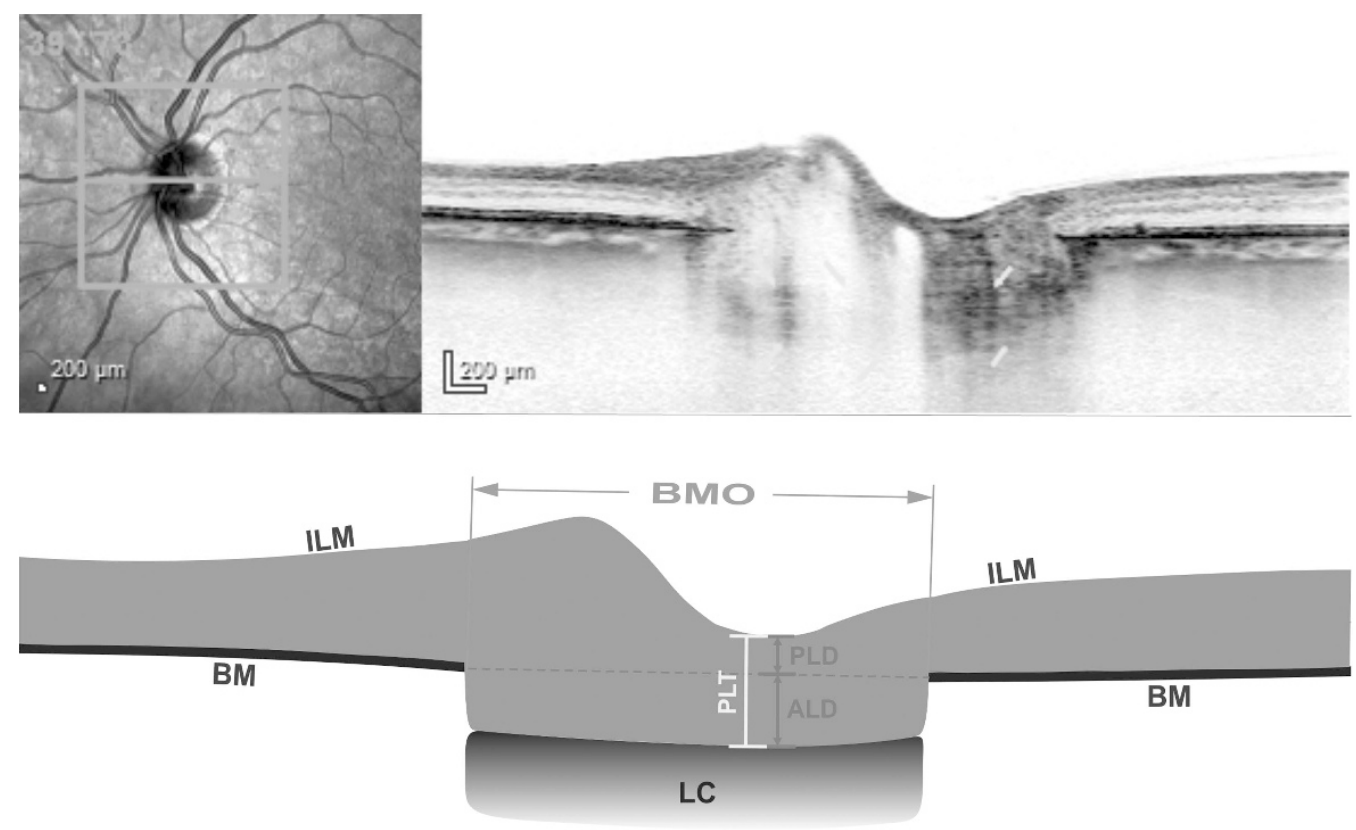

Figure 1 Optic nerve head parameters. Top: original OCT image. Bottom: Schematic illustration of measurements: internal limiting membrane (ILM), Bruch's membrane (BM), connection line between two BM terminations (Bruch's membrane opening (BMO); dash red line), lamina cribrosa (LC); anterior laminar depth (ALD); prelaminar depth (PLD); Prelaminar tissue thickness (PLT; yellow line). A full colour version of this figure is available at the Eye journal online.

(direction normal to the $\mathrm{BMO}$ reference plane) between the internal limiting membrane surface and anterior laminar surface. For this measurement in a central B-scan, the distance from the maximally depressed point of the optic nerve surface (in a temporal direction) to the level of anterior border of LC was measured. Only the temporally depress point was selected because the elevated nasal points were often caused by vessel trunk of which the shadow obscured the LC. ${ }^{14}$ The perpendicular distance from the $\mathrm{BMO}$ reference plane to the anterior laminar surface was termed the ALD (Figure 1). In the last step, the inner tip of the BMO was marked on all raster scans. The location of the inner tip of the BMO on B-scans was projected onto SD-OCT en face infrared images and these locations were connected together to calculate the $\mathrm{BMO}$ area (Figure 2). ${ }^{15}$ All images were analyzed by two specialists (SM and MAF) in two different sessions. To evaluate the intra-observer and inter-observer reproducibility of the $\mathrm{ONH}, 15$ randomly selected EDIOCT B-scans from 15 eyes were evaluated. The absolute agreement of a single observer's measurements and the average of the measurements conducted by the 2 observers were calculated using the intra-class correlation coefficient (ICC) via a two-way mixed effect model. Finally, the clinical optic disc contour was marked twice at the inner border of the scleral ring on infrared images while viewing color photographs of the optic disc by an experienced clinician (MAF). ${ }^{4,16}$ The OCT software calculated the optic disc area parameter. The average of the two values was recorded as the optic disc area. The clinical optic disc area was measured as a risk factor for NAION in comparison with the BMO area.

\section{Statistical methods}

Data are presented as mean and SD. To compare the demographic characteristics of the patients, we ignored the unaffected fellow eye of the NAION patients and compared the three groups by $t$-test and $\chi^{2}$-test. Then, we employed a linear mixed model to evaluate the $\mathrm{ONH}$ parameters between groups using the Bonferroni correction for multiple comparisons and inter-eye correlation. In the last step, another linear mixed model was used to adjust the effect of age, gender, and axial length in our analysis. Multivariable linear regression model also was used to evaluate the relationship between the prelaminar thickness with cup-disc-ratio, age, gender, and axial length. The SPSS software (IBM Corp. Released 2013. IBM SPSS Statistics for Windows, Version 22.0. Armonk, NY, USA: IBM Corp.) was used for all statistical analyses. $P$-values $<0.05$ were considered significant.

\section{Results}

Thirty-five patients with chronic unilateral NAION and thirty-five healthy eyes were initially enrolled in this study. Six patients with poor image quality were 

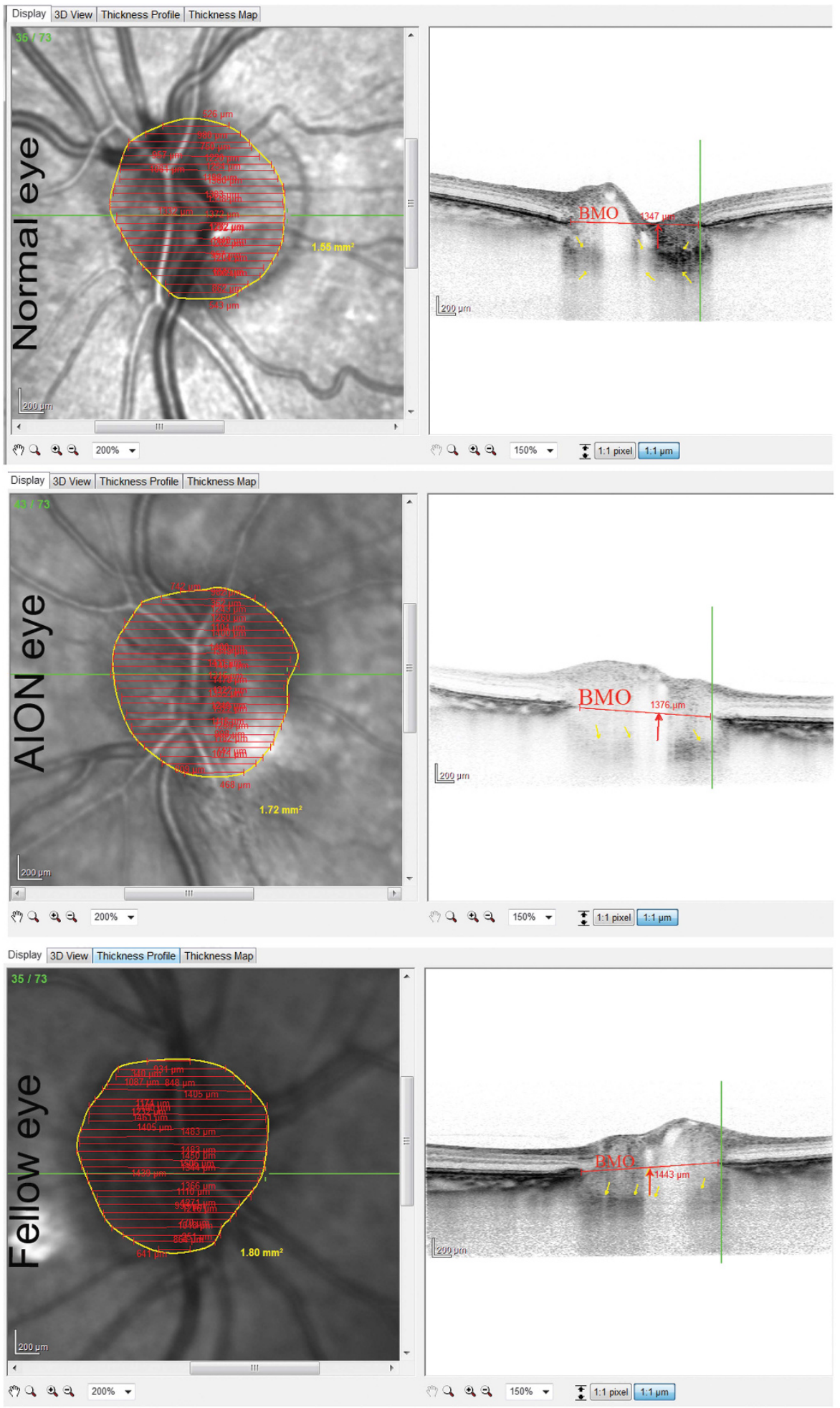

Figure 2 Upper row: (left) An infrared image of the normal optic nerve used to measure Bruch's membrane opening (BMO) area. Several BMO lines were marked on all optic nerve head raster scans (right). These lines on B-scan were projected onto en face infrared images. Ends of lines connected and BMO area was measured. In this case BMO area is $1.55 \mathrm{~mm}^{2}$. A raster line across the center of optic nerve shows BMO distance (Right; red line), anterior laminar depth (red arrow), and anterior and posterior borders of lamina cribrosa (yellow arrows). Red numbers shows central BMO distances. Middle row: (left) the BMO area is $1.72 \mathrm{~mm}^{2}$ in an optic nerve with a history of non-arteritic anterior ischemic optic neuropathy (NAION) 5 months ago. Prelaminar tissue is thicker than normal eyes (Right). Lower row: The BMO area is $1.80 \mathrm{~mm}^{2}$ in an unaffected optic nerve head of the same patient with NAION with thick prelaminar tissue. 
Table 1 Demographic and baseline characteristics of patients with unilateral non-arteritic anterior ischemic optic neuropathy (NAION) and normal subjects

\begin{tabular}{lcccc}
\hline Parameter & \multicolumn{3}{c}{ Group } & \multirow{2}{*}{ P-value } \\
\cline { 2 - 4 } & AION & Fellow eye & Normal \\
\hline Sex (male) & $16(48.5 \%)$ & $16(48.5 \%)$ & $13(41.9 \%)$ & $0.11 \pm 0.18$ \\
LogMAR & $1.1 \pm 1.03$ & $0.07 \pm 0.12$ & $22.97 \pm 1.34$ & $<0.001^{\mathrm{b}}$ \\
Axial length & $22.69 \pm 1$ & $22.71 \pm 0.99$ & $0.2 \pm 0.9$ & $0.421^{\mathrm{b}}$ \\
Mean deviation & $-19.43 \pm 8.8$ & $-1.1 \pm 2.83$ & $99.97 \pm 12.05$ & $<0.001^{\mathrm{b}}$ \\
Average RNFL & $60.64 \pm 19.24$ & $102.36 \pm 10.26$ & $<0.001^{\mathrm{b}}$ \\
\hline
\end{tabular}

${ }^{a}$ Based on Chi-Square test, comparison of AION and Normal group. ${ }^{b}$ Based on Linear Mixed Model (LMM).

excluded. Therefore, 66 eyes of 33 patients with unilateral NAION and 31 eyes of 31 healthy normal subjects were included in this study.

The basic characteristics of the study participants are listed in Table 1. There were no statistically significant differences in age, gender and axial length between the NAION and control eyes. The mean deviation of the visual field was $-19.43 \pm 8.8 \mathrm{~dB}$ in the NAION eyes. Mean cup-to-disc ratio was $0.14 \pm 0.10,0.10 \pm 0.09$, and $0.31 \pm 0.16$ in NAION and unaffected fellow eyes, and control eyes, respectively. Cup-to-disc ratio in NAION and fellow eyes was smaller than that of control eyes $(P<0.001)$. The mean $\mathrm{pRNFL}$ of the NAION group was significantly thinner than that of the healthy control group and the unaffected fellow eyes $(P=0.001$ and $P=0.03$, respectively).

Intra-observer and inter-observer reproducibility ranged from an ICC of 0.852 to 0.999 for various $\mathrm{ONH}$ parameters (Table 2).

Linear mixed model analysis showed a statistical difference in the disc area but there were no significant differences in the superior, inferior, and central ALD, and the BMO distance and area among the three groups (Table 3). Although the clinical optic disc area was smaller in NAION eyes $\left(1.94 \pm 0.35 \mathrm{~mm}^{2}\right)$ and fellow eyes $\left(1.87 \pm 0.37 \mathrm{~mm}^{2}\right)$ than control eyes $\left(2.23 \pm 0.43 \mathrm{~mm}^{2}\right.$; $P=0.006)$, the BMO area was similar in NAION eyes $\left(1.89 \pm 0.33 \mathrm{~mm}^{2}\right)$, fellow eyes $\left(1.85 \pm 0.35 \mathrm{~mm}^{2}\right)$, and control eyes $\left(1.88 \pm 0.37 \mathrm{~mm}^{2} ; P>0.99\right)$. The central, inferior, and superior ALD of the NAION group and unaffected fellow eyes was similar to that of the healthy control group.

Linear mixed model analysis showed statistical differences in the central prelaminar depth and thickness between the groups.

In the NAION group, the mean central prelaminar depth was $-110 \pm 61 \mu \mathrm{m}$, which was thinner than that of the unaffected fellow eyes $(-167 \pm 172 \mu \mathrm{m}$, $P=0.03$ ). The mean prelaminar depth in the control group was $81 \pm 129 \mu \mathrm{m}$, and there was a significant difference between the control group, the NAION
Table 2 Interclass coefficient correlation (ICC) for optic nerve head measurements

\begin{tabular}{lcc}
\hline & $\begin{array}{c}\text { Intra-observer ICC } \\
(95 \% \text { CI })\end{array}$ & $\begin{array}{c}\text { inter-observer ICC } \\
(95 \% \text { CI })\end{array}$ \\
\hline BMO distance & $0.992(0.975-0.998)$ & $0.976(0.916-0.991)$ \\
BMO area & $0.943(0.909-0.977)$ & $0.899(0.852-0.931)$ \\
Prelaminar & $0.997(0.989-0.999)$ & $0.977(0.919-0.991)$ \\
thickness & & \\
CALD & $0.974(0.916-0.992)$ & $0.943(0.816-0.972)$ \\
SALD & $0.998(0.993-0.999)$ & $0.962(0.923-0.993)$ \\
IALD & $0.944(0.757-0.987)$ & $0.890(0.711-0.959)$ \\
\hline
\end{tabular}

Abbreviations: cALD, central anterior laminar depth; sALD, superior anterior laminar depth; iALD, inferior anterior laminar depth; BMO, Bruch's membrane opening; NAION, Non-arteritic ischemic optic neuropathy.

group, and the fellow eyes (both $P<0.001$ ), indicating that the prelaminar depth was significantly elevated in both NAION and fellow eyes (with minus sign) compared to control eyes, which was shallow (Figure 2).

The mean prelaminar tissue thickness of the NAION group was $445 \pm 176 \mu \mathrm{m}$ which was thinner than the unaffected eyes (mean, $539 \pm 227 \mu \mathrm{m}, P=0.004$ ), but both were thicker than the prelaminar tissue of the normal subjects (mean $243 \pm 145 \mu \mathrm{m}, P=0.001$ and $P<0.001$, respectively).

In all study eyes cup-to-disc ratio was significantly correlated central prelaminar thickness $(r=-0.37$, $P<0.001)$. Multivariate linear regression analysis that controlled for the effect of potential confounders (age, gender, and axial length) showed that 0.1 decrease in cupto-disc ratio was associated with $48.11 \mu \mathrm{m}$ increase in central prelaminar thickness $(P<0.001)$.

\section{Discussion}

Accurate characterization of the $\mathrm{ONH}$ structure and the potential risk factors of ischemic event would be of clinical use in NAION. In the present study, we used the EDI SD-OCT quantification to compare the $\mathrm{ONH}$ parameters between old unilateral NAION eyes, 
unaffected fellow eyes, and control eyes. The prelaminar depth was significantly in NAION and fellow eyes different (both with a minus sign) when compared to the control eyes (with plus sign). The prelaminar tissue was $110 \pm 61 \mu \mathrm{m}$ and $167 \pm 172 \mu \mathrm{m}$ above the BMO plane on average in NAION and their unaffected fellow eyes, respectively. The prelaminar depth was $81 \pm 129 \mu \mathrm{m}$ under the $\mathrm{BMO}$ in normal eyes. In other words, the internal limiting membrane dipped below the BMO reference plane in normal eyes and less likely in NAION and their unaffected eyes. Interestingly, because of excess neural and/or glial tissue ${ }^{17}$ in the $\mathrm{ONH}$ and prelaminar depth elevation in NAION, the prelaminar depth was still greater in NAION when compared to control eyes rather than prelaminar neural tissue thinning due to nerve fiber layer loss in NAION patients. We also observed a thick prelaminar tissue not only in NAION eyes but also in the unaffected fellow eyes of NAION patients compared to healthy eyes. Prelaminar depth was compared between the unaffected fellow eyes of the patients with NAION and normal control eyes to assess the premorbid characteristics of eyes with NAION. Considering the presence of a thick prelaminar tissue in both the involved and uninvolved eye of patients with NAION, one can postulate this as a primary event rather than a secondary phenomenon. In other words, because the contralateral unaffected eyes of the patients with unilateral NAION could be involved, one may suppose that the thick prelaminar tissue may have been present before the onset of the disease in our NAION patients.

Another contributing factor for developing NAION are the cupless optic nerve. ${ }^{2-6}$ We also found smaller cup-todisc ratio in NAION and their unaffected eyes compared to control eyes and 0.1 decrease in cup-to-disc ratio was associated with $48.11 \mu \mathrm{m}$ increase in prelaminar thickness. Therefore, thick prelaminar tissue might stem from the original observation that the cup-to-disc ratio is smaller in these patients.

Previous studies have shown small optic disc diameter in affected and unaffected eyes of NAION. ${ }^{16,18}$ Hayreh et al 'hypothesized' that the optic discs of NAION patients tended to have smaller openings in their Bruch's membrane than the general population, resulting in crowding of the optic disc. However, the BMO area was similar in NAION eyes $\left(1.89 \pm 0.33 \mathrm{~mm}^{2}\right)$, fellow eyes $\left(1.85 \pm 0.35 \mathrm{~mm}^{2}\right)$, and control eyes $\left(1.88 \pm 0.37 \mathrm{~mm}^{2}\right)$ and we did not find any differences in the BMO diameter and $\mathrm{BMO}$ area between the three groups. Similarly, Sibony et $a l^{19}$ did not find any differences in the horizontal diameter of the neural canal opening between NAION and normal eyes. A recent study also did not find any differences in the BMO area between NAION and control subjects. ${ }^{20}$ The discrepancy between the optic disc size in previous studies and the BMO area in our study could be 
explained by the fact that the BMO does not always coincide with the clinical optic disc margin. The SD-OCT structure most commonly coinciding with the clinically identified disc margin was a location on the Bruch's membrane with a border tissue beneath (when the innermost edges of Bruch's membrane and border tissue did not coincide) and the termination of Bruch's membrane infrequently colocalized with the optic disc margin. ${ }^{12,21}$

Therefore, a thicker prelaminar tissue and its associated small cup-to-disc ratio (but not a small BMO area) in NAION and fellow eyes contribute to the structural optic nerve crowding as they pass through a restricted space in the optic disc. This 'crowded' morphologic appearance is believed to predispose the optic nerve head to ischemia because of presumed structural crowding of the approximately 1 million axons. ${ }^{18}$

Recent study evaluated the influence of acute NAION on the LC position and prelaminar thickness. They observed a significant thickening of the prelaminar thickness with backward movement of the LC and enlargement of BMO diameter in the acute phase, followed by significant prelaminar thinning and forward LC movement at 2 and 6 months. However, they did not include normal controls and did not measure BMO area. ${ }^{13}$

In this study, we also found that the prelaminar tissue thickness decreased in NAION eyes compared to unaffected fellow eyes. Similarly, Henkind et $a l^{22}$ demonstrated the atrophy of the prelaminar tissue in the histological findings of a 67-year-old woman with arterial anterior ischemic optic neuropathy. The loss of retinal ganglion cells and their axon in NAION eyes might cause prelaminar thinning. ${ }^{23,24}$ Similar to ischemic optic neuropathy, compressive optic neuropathy accompanies thinning of the mean prelaminar tissue thickness without laminar remodeling. ${ }^{25}$ There were no significant differences in the superior, inferior, and central ALD, among the three groups in our study and it has been shown that the absence of laminar deformation determines the feature of nonglaucomatous optic neuropathy. ${ }^{12,26}$

Our study had several limitations. Our sample size was small but it was in line with previous studies. However, given the very significant difference in thickness between the groups, the overall results of the study could be generalized. On the other hand, visualizing LC, on the other hand, could be more difficult in NAION patients with a thick prelaminar tissue, resulting in poor resolution of the LC layer. Also, we used the BMO as a reference plane in one B-scan for measuring the laminar and prelaminar depth and we did not create our reference plane based on all BMO points. Therefore, it is possible that laminar measurements in a single plane were not exactly perpendicular to the best fit plane of the BMO anatomy. However, this possible error could occur to all three groups to the same degree and our comparisons between groups were not therefore affected. In addition, since the Bruch's membrane lies on the anterior surface of the choroid, the BMO position may be influenced by the choroidal thickness. ${ }^{27}$ In fact, There are reports of an increased peripapillary choroidal thickness in the NAION patients, ${ }^{16,20}$ indicating that the laminar depth under the BMO might be overestimated. However, it is likely that the measurement of the structures anterior (above) to the BMO such as the prelaminar depth and prelaminar thickness was not affected in NAION patients. ${ }^{27}$

In conclusion, we found thinning of the peripapillary nerve fiber layer tissues without important laminar involvement in NAION eyes. The prelaminar depth and thickness were greater in NAION and unaffected fellow eyes when compared to the control eyes.

\section{Summary}

What was known before

- A clinically smaller optic disc area, a crowded optic nerve, and a smaller cup-to-disc ratio were considered as the main risk factors for development of non-arteritic anterior ischemic optic neuropathy (NAION).

What this study adds

- A thicker prelaminar tissue and its associated small cupto-disc ratio (but not a small BMO area) in NAION and fellow eyes contribute to the structural optic nerve crowding as they pass through a restricted space in the optic disc.

\section{Conflict of interest}

The authors declare no conflict of interest.

\section{References}

1 Arnold C. Ischemic optic neuropathyMiller NR, Newman NJ, Biousse V. Clinical Neuro-Ophthalmology. 6th edn Lippincott Williams \& Wilkins: Philadelphia: 2005; Vol. 1, 349-384.

2 Doro S, Lessell S. Cup-disc ratio and ischemic optic neuropathy. Arch Ophthalmol 1985; 103: 1143-1144.

3 Beck RW, Servais GE, Hayreh SS. Anterior ischemic optic neuropathy. IX. Cup-to-disc ratio and its role in pathogenesis. Ophthalmology 1987; 94: 1503-1508.

4 Saito H, Tomidokoro A, Tomita G, Araie M, Wakakura M. Optic disc and peripapillary morphology in unilateral nonarteritic anterior ischemic optic neuropathy and age and refraction matched normals. Ophthalmology 2008; 115: 1585-1590.

5 Contreras I, Rebolleda G, Noval S, Muñoz-Negrete FJ. Optic disc evaluation by optical coherence tomography in 
nonarteritic anterior ischemic optic neuropathy. Invest Ophthalmol Vis Sci 2007; 48: 4087-4092.

6 Hayreh SS, Zimmerman MB. Nonarteritic anterior ischemic optic neuropathy: refractive error and its relationship to cup/disc ratio. Ophthalmology 2008; 115: 2275-2281.

7 Yang D, Fu J, Hou R, Liu K, Jonas JB, Wang H et al. Optic neuropathy induced by experimentally reduced cerebrospinal fluid pressure in monkeys. Invest Ophthalmol Vis Sci 2014; 55: 3067-3073.

8 Ing E, Ivers KM, Yang H, Gardiner SK, Reynaud J, Cull G et al. Cupping in the monkey optic nerve transection model consists of prelaminar tissue thinning in the absence of posterior laminar deformation. Invest Ophthalmol Vis Sci 2016; 57: 2598-2611.

9 Park SC, De Moraes CG, Teng CC, Tello C, Liebmann JM, Ritch R. Enhanced depth imaging optical coherence tomography of deep optic nerve complex structures in glaucoma. Ophthalmology 2012; 119: 3-9.

10 Park HY, Park CK. Diagnostic capability of lamina cribrosa thickness by enhanced depth imaging and factors affecting thickness in patients with glaucoma. Ophthalmology 2013; 120: 745-752.

11 Chauhan BC, Burgoyne CF. From clinical examination of the optic disc to clinical assessment of the optic nerve head: a paradigm change. Am J Ophthalmol 2013; 156: 218-227.e2.

12 Fard MA, Afzali M, Abdi P, Chen R, Yaseri M, Azaripour E et al. Optic nerve head morphology in nonarteritic anterior ischemic optic neuropathy compared to open-angle glaucoma. Invest Ophthalmol Vis Sci 2016; 57: 4632-4640.

13 Rebolleda G, García-Montesinos J, De Dompablo E, Oblanca N, Muñoz-Negrete FJ, González-López JJ. Bruch's membrane opening changes and lamina cribrosa displacement in nonarteritic anterior ischaemic optic neuropathy. $\mathrm{Br}$ J Ophthalmol 2017; 101: 143-149.

14 Lee EJ, Kim T-W, Weinreb RN. Reversal of lamina cribrosa displacement and thickness after trabeculectomy in glaucoma. Ophthalmology 2012; 119: 1359-1366.

15 Miri MS, Abràmoff MD, Lee K, Niemeijer M, Wang JK, Kwon YH et al. Multimodal segmentation of optic disc and cup from SD-OCT and color fundus photographs using a machine-learning graph-based approach. IEEE Trans Med Imaging 2015; 34: 1854-1866.

16 Fard MA, Abdi P, Kasaei A, Soltani Mogaddam R, Afzali M, Moghimi S. Peripapillary choroidal thickness in nonarteritic anterior ischemic optic neuropathy. Invest Ophthalmol Vis Sci 2015; 56: 3027-3033.

17 Jonas JB, Hayreh SS, Tao Y, Papastathopoulos KI, Rensch F. Optic nerve head change in non-arteritic anterior ischemic optic neuropathy and its influence on visual outcome. PLoS One 2012; 7: e37499 386.

18 Miller NR, Arnold AC. Current concepts in the diagnosis, pathogenesis and management of nonarteritic anterior ischaemic optic neuropathy. Eye (Lond) 2015; 29: 65-79.

19 Sibony P, Kupersmith MJ, Rohlf FJ. Shape analysis of the peripapillary RPE layer in papilledema and ischemic optic neuropathy. Invest Ophthalmol Vis Sci 2011; 52: 7987-7995.

20 Nagia L, Huisingh C, Johnstone J, Kline LB, Clark M, Girard MJ et al. Peripapillary pachychoroid in nonarteritic anterior ischemic optic neuropathy. Invest Ophthalmol Vis Sci 2016; 57: 4679-4685.

21 Reis AS, Sharpe GP, Yang H, Nicolela MT, Burgoyne CF, Chauhan BC. Optic disc margin anatomy in patients with glaucoma and normal controls with spectral domain optical coherence tomography. Ophthalmology 2012; 119: 738-747.

22 Henkind P, Charles NC, Pearson J. Histopathology of ischemic optic neuropathy. Am J Ophthalmol 1970; 69: 78-90.

23 Fard MA, Ebrahimi KB, Miller NR. RhoA activity and postischemic inflammation in an experimental model of adult rodent anterior ischemic optic neuropathy. Brain Res 2013; 1534: 76-86.

24 Akbari M, Abdi P, Fard MA, Afzali M, Ameri A, YazdaniAbyaneh A et al. Retinal ganglion cell loss precedes retinal nerve fiber thinning in nonarteritic anterior ischemic optic neuropathy. I Neuroophthalmol 2016; 36: 141-146.

25 Hata M, Miyamoto K, Oishi A, Makiyama Y, Gotoh N, Kimura $\mathrm{Y}$ et al. Comparison of optic disc morphology of optic nerve atrophy between compressive optic neuropathy and glaucomatous optic neuropathy. PLoS One 2014; 9: e112403.

26 Burgoyne $\mathrm{C}$. The morphological difference between glaucoma and other optic neuropathies. J Neuroophthalmol 2015; 35(Suppl): S8-S21.

27 Johnstone J, Fazio M, Rojananuangnit K, Smith B, Clark M, Downs C et al. Variation of the axial location of Bruch's membrane opening with age, choroidal thickness, and race. Invest Ophthalmol Vis Sci 2014; 55: 2004-2009. 